\title{
Noise-enhanced classical and quantum capacities in communication networks
}

\author{
Filippo Caruso, Susana F. Huelga, and Martin B. Plenio \\ Institut für Theoretische Physik, Albert-Einstein-Allee 11, Universität Ulm, D-89069 Ulm, Germany
}

\begin{abstract}
The unavoidable presence of noise is thought to be one of the major problems to solve in order to pave the way for implementing quantum information technologies in realistic physical platforms. However, here we show a clear example in which noise, in terms of dephasing, may enhance the capability of transmitting not only classical but also quantum information, encoded in quantum systems, through communication networks. In particular, we find analytically and numerically the quantum and classical capacities for a large family of quantum channels and show that these information transmission rates can be strongly enhanced by introducing dephasing noise in the complex network dynamics.
\end{abstract}

Introduction.- An important obstacle for the development of quantum communication technologies is the difficulty of transmitting quantum information over noisy quantum communication channels, recovering and refreshing it at the receiver side, and then storing it in a reliable quantum memory [1, 2]. This concerns both point-to-point communication as well as more complex quantum networks consisting of several nodes. These operations are necessary as the unavoidable presence of noise during transmission via a quantum channel and its processing at the receiver's end is generally expected to degrade the transmission quality. It was pointed out however, that noise may in fact have a positive influence on sustaining quantum correlations [3]. This motivated some early explorations of the potentially beneficial effects that noise may have on information transmission through quantum channels [4]. It was not possible however to compute capacities in those examples and attention focussed on related quantities that were furthermore restricted to certain input states. As a result, no firm conclusion could be drawn from these considerations. Recently though, it was realised in a different context that noise may have a positive impact on transport phenomena in complex networks. In fact, it was found that excitation energy transfer (EET) in light harvesting complexes during photosynthesis can benefit considerably from the presence of dephasing noise [5, 6]. Here, it is the intricate interplay of noise and quantum coherence that explains the remarkable efficiency, well above $90 \%$, for EET in light harvesting complexes during photosynthesis whereas noise-free systems exhibit efficiencies of around $50 \%$ only [7]. Motivated by these results, we study the scenario of a realistic communication network, subjected to a noisy evolution, and derive analytically and numerically the channel capacities. We will demonstrate that both classical and quantum channel capacities can increase thanks to the presence of dephasing noise. Remarkably, in the case of quantum capacities, the presence of noise may lead to a finite quantum capacity where the noiseless system has vanishing capacity.

The Model.- We consider a generic complex network of $N$ vertices, in which each site represents a two-level quantum system (qubit). Suppose that the sender of the message, Alice (A), wants to transmit a message to the receiver, Bob (B), by using such quantum network. The communication protocol can be the following: i) Alice applies a swap operation in

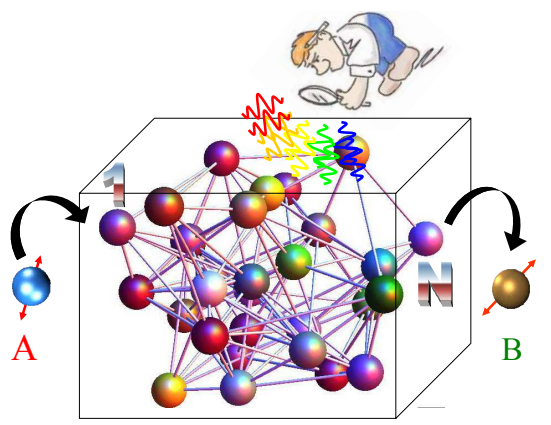

FIG. 1. Communication network: on one side, Alice sends a message encoded in the qubit 1 , while the rest of the network is prepared in its ground state. On the other side, after some noisy evolution of the network state, Bob tries to recover Alice's message decoding his output qubit state in the site $N$.

order to set the unknown initial state $\rho_{A}$ in the site 1 , while the rest of the network is initially prepared in the ground state $|0 \ldots 0\rangle$, ii) then they let the network state evolve under some quantum noisy evolution, iii) at time $t_{\text {out }}$, Bob tries to recover the information sent by Alice through some decoding procedures applied to his output state $\rho_{B}\left(t_{\text {out }}\right)$, which corresponds to the reduced density operator for the $N$ th qubit (up to a local unitary transformation). Mathematically, at each time $t_{\text {out }}$, one can describe this process as a completely positive and trace-preserving (CPTP) quantum channel of the form

$$
\rho_{B}\left(t_{\text {out }}\right) \equiv \mathcal{E}\left(\rho_{A}\right)=\operatorname{Tr}_{E}\left[U\left(t_{\text {out }}\right)\left(\rho_{A} \otimes \rho_{E}\right) U\left(t_{\text {out }}\right)^{\dagger}\right]
$$

where $\rho_{E}$ is the initial state of some environment and $U\left(t_{\text {out }}\right)$ is the unitary evolution of system + environment for a time $t_{\text {out }}$. For instance, in the case of an Hamiltonian evolution of the network, $\rho_{E}$ is the ground state of all the qubits $2, \ldots, N$ and $U\left(t_{\text {out }}\right)=\exp \left[-i H t_{\text {out }} / \hbar\right]$ is the unitary evolution operator associated to the Hamiltonian $H$. In this case, following Ref. [8], if the Hamiltonian commutes with the Pauli operator $\sigma_{z}$, i.e. the number of qubits in the state $|1\rangle$ is constant in time, at each time step $t_{\text {out }}$ the corresponding quantum map is an amplitude damping channel $\mathcal{D}(\eta)$, with the damping coefficient given by $\eta=\left\langle N\left|U\left(t_{\text {out }}\right)\right| 1\right\rangle$, with the convention that $|j\rangle$ denotes the state in which all the qubits are in the state $|\downarrow\rangle$, except the qubit $j$ in the state $|\uparrow\rangle$. In particular, Ref. [8] considered a spin chain sub- 
jected to an Heisenberg Hamiltonian evolution and in this context derived the channel capacities. Here, we investigate a noisy evolution of a network of $N$ qubits, in which, for instance, some pure dephasing noise is present in the dynamics and, as shown later, will play a key role in the information transfer rates of the corresponding communication channel. For simplicity, we will consider the following Hamiltonian, $H=\sum_{j=1}^{N} \hbar \omega_{j} \sigma_{j}^{+} \sigma_{j}^{-}+\sum_{j \neq l} \hbar v_{j, l}\left(\sigma_{j}^{-} \sigma_{l}^{+}+\sigma_{j}^{+} \sigma_{l}^{-}\right)$, and a local Lindblad term that takes into account the dephasing caused by some surrounding environment, i.e., $\mathcal{L}_{\text {deph }}(\rho)=$ $\sum_{j=1}^{N} \gamma_{j}\left[-\left\{\sigma_{j}^{+} \sigma_{j}^{-}, \rho\right\}+2 \sigma_{j}^{+} \sigma_{j}^{-} \rho \sigma_{j}^{+} \sigma_{j}^{-}\right]$, with $\sigma_{j}^{+}\left(\sigma_{j}^{-}\right)$being the raising and lowering operators for site $j, \hbar \omega_{j}$ being the local site excitation energy, $v_{k, l}$ denoting the hopping rate of an excitation between the sites $k$ and $l$, and $\gamma_{j}$ being the dephasing rate at the site $j$. Let us point out, however, that the following results must be valid for any Hamiltonian, provided that it commutes with the operator $\sigma_{z}$, i.e. preserving the number of 'excitations' in the network evolution, and quite reasonably for other forms of noise, also beyond the Markovian approximation. Generalizing Ref. [8], it is possible to show that, at each time $t_{\text {out }}$, the corresponding CPTP quantum channel is of the following form:

$\mathcal{E}(\eta, s): \rho_{A}=\left(\begin{array}{cc}p & \gamma \\ \gamma^{*} & 1-p\end{array}\right) \rightarrow \rho_{B}=\left(\begin{array}{cc}\eta p & \sqrt{\eta s} \gamma \\ \sqrt{\eta s} \gamma^{*} & 1-\eta p\end{array}\right)$

with $p$ a real number in the range $[0,1]$ and $\gamma$ a complex number such that $|\gamma|^{2} \leq p(1-p)$. The channel is completely defined by two time-dependent parameters: i) $\eta \in[0,1]$ describing the population damping, and ii) $s \in[0,1]$ includes the decoherence effects. On one hand, because of the linearity of a quantum channel, $\eta(t)$ corresponds to the population of site $N$ at time $t$ when $\rho_{A}=|1\rangle\langle 1|$, i.e. one excitation is initially in the site 1 . On the other hand, $s$ is generally a complicate time-dependent function of all the parameters involved in the noisy evolution and later will be determined numerically considering a generic input qubit state. Note also that $s$ can be considered a real number because any phase $e^{i \theta}$ can be eliminated applying a local unitary transformation, i.e. $|1\rangle \rightarrow e^{-i \theta}|1\rangle$, and the quantities analyzed later are invariant under such operations. Besides, it can be easily shown that the map in (2) is equivalent to a consecutive application of an amplitude damping channel $\mathcal{D}(\eta)$ and a phase-flip channel $\mathcal{N}(s)$ (unitarily equivalent to a dephasing channel), changing the phase of the state $|1\rangle$, i.e. $|1\rangle \rightarrow-|1\rangle$, with probability $(1-\sqrt{s}) / 2$. In other words, one has $\mathcal{E}(\eta, s)=\mathcal{D}(\eta) \circ \mathcal{N}(s)=\mathcal{N}(s) \circ \mathcal{D}(\eta)$. In general, a CPTP quantum channel can be also represented in an elegant form known as operator-sum (or Kraus) representation [1], i.e. $\mathcal{E}\left(\rho_{A}\right)=\sum_{k} A_{k} \rho_{A} A_{k}^{\dagger}$, where the so-called Kraus operators $A_{k}$ satisfy the condition $\sum_{k} A_{k}^{\dagger} A_{k}=\mathbb{1}$. In particular, these operators for the map in (2) are given by $A_{1}=\operatorname{diag}(0, \sqrt{s \eta})$, $A_{2}=\operatorname{anti-\operatorname {diag}}(0, \sqrt{1-\eta})$, and $A_{3}=\operatorname{diag}(0, \sqrt{(1-s) \eta})$. In fact, one can show that $\mathcal{E}(\rho)=\sum_{k=1}^{3} A_{k} \rho_{A} A_{k}^{\dagger}$ corresponds exactly to $\rho_{B}$ as in Eq. (2). In the following, we will analyze the capability of the channel in (2) for transmit-

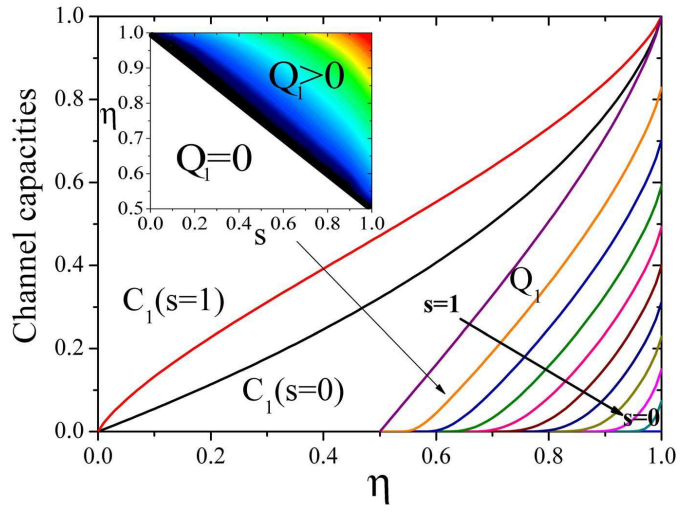

FIG. 2. Classical and quantum capacities, $C_{1}(\mathcal{E}(\eta, s))$ and $Q_{1}(\mathcal{E}(\eta, s))$, versus $\eta$ and $s$. Inset: contour plot for $Q_{1}(\mathcal{E}(\eta, s))$ as a function of $s$ and $\eta$ in a color gradient representation where red corresponds to 1 and black to vanishing values of $Q_{1}$. The quantum capacity is always zero for $\eta \leq 1 / 2$ and any $s$.

ting classical and quantum information asymptotically undisturbed, i.e. with a vanishing error probability in the limit of long messages and with some optimal encoding and decoding schemes, by calculating, respectively, the classical and quantum channel capacities and some related figures of merit.

Classical Capacity.- The so-called 'one-shot' or product state classical capacity of a quantum channel is obtained maximizing the Holevo information [9], i.e.

$$
C_{1}(\mathcal{E})=\max _{\xi_{k}, \rho_{k}}\left[S\left(\sum_{k} \xi_{k} \mathcal{E}\left(\rho_{k}\right)\right)-\sum_{k} \xi_{k} S\left(\mathcal{E}\left(\rho_{k}\right)\right)\right]
$$

where the maximum is taken over all probability distributions $\xi_{k}$ and collections of density operators $\rho_{k}$ and $S(\rho)=$ $-\operatorname{Tr}\left[\rho \log _{2} \rho\right]$ is the von Neumann entropy of the state $\rho$. This is the classical capacity with unentangled encodings while a full maximization over multiple channel uses would provide the unrestricted classical capacity of a quantum channel. For simplicity, we will focus here on the case of unentangled encodings which yields lower bounds for the unrestricted classical capacity of a quantum channel. Notice, however, that the classical capacity with entangled encodings is a monotonic increasing function of $\eta$ (by the bottleneck inequality), and then, in the presence of a dephasing-induced enhancement of the population transfer $(\eta)$, also the full classical capacity is enhanced. Now, we calculate $C_{1}$ for the family of quantum channels in (2). In the case $s=1$, the channel reduces to $\mathcal{D}(\eta)$, whose channel capacities were studied in Ref. [8]. Here, first we will solve analytically the case of $s=0$, which will be relevant in the example below. Let us consider an ensemble $\left\{\xi_{k}, \rho_{k}\right\}$, with $\rho_{k}$ of the form of $\rho_{A}$ in Eq. (2), with parameters $p_{k}$ and $\gamma_{k}$. The ensemble average state will be a state $\rho$ with coefficients $p$ and $\gamma$ such that $p=\sum_{k} \xi_{k} p_{k}$ and $\gamma=\sum_{k} \xi_{k} \gamma_{k}$. In the case of $s=0$, it can be easily shown that the Holevo information reduces to $H_{2}(\eta p)-\sum_{k} \xi_{k} H_{2}\left(\eta p_{k}\right)$, with $H_{2}$ being the binary entropy function defined as $H_{2}(x)=-x \log _{2} x-(1-x) \log _{2}(1-x)$. 


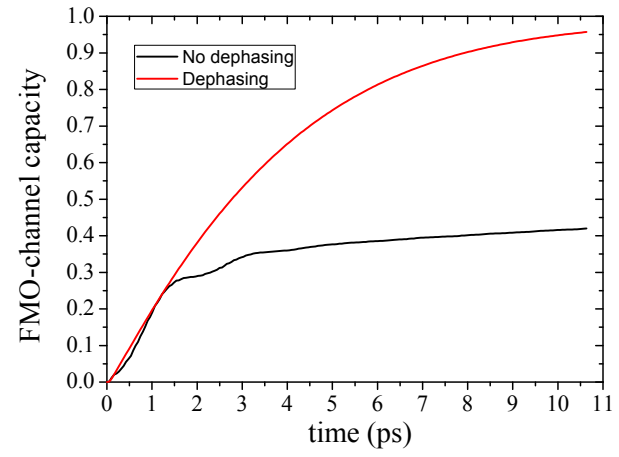

FIG. 3. Classical capacity of the FMO-complex quantum channel, $C_{1}\left(\mathcal{E}\left(p_{\text {sink }}(t), 0\right)\right)$, vs. time, in the absence and presence of dephasing. There is a clear remarkable enhancement of $C_{1}$ in the case of pure dephasing noise.

By exploiting the concavity property of $\mathrm{H}_{2}$, one finds that $C_{1}(\mathcal{E}(\eta, 0)) \leq \max _{p \in[0,1]}\left[H_{2}(\eta p)-p H_{2}(\eta)\right]$. The last step of this analytical calculation consists of showing that this upper bound is actually tight and can be obtained with the following optimal ensemble defined by the coefficients $\xi_{1}=p$ and generic $\xi_{i}$ such that $\sum_{k} \xi_{k}=1$, with $p_{1}=1$ and $p_{i}=0$, for $i=1, \ldots d$, where $d$ is the generic dimension of the ensemble $\left\{\xi_{k}, \rho_{k}\right\}$. Therefore, we find that

$$
C_{1}(\mathcal{E}(\eta, 0))=H_{2}(\eta \bar{p})-\bar{p} H_{2}(\eta)
$$

where $\bar{p}=\left[(1-\eta)^{(\eta-1) / \eta}+\eta\right]^{-1}$ is the optimal value of $p$, which we find analyzing that function analytically - see Fig. 2. Notice that, because of the composition law above, one has also that $\mathcal{E}\left(\eta, s_{1}\right)=\mathcal{E}\left(\eta, s_{2}\right) \circ \mathcal{N}\left(s_{1} / s_{2}\right)$ for $s_{1}<s_{2}$. Hence, by the bottleneck inequality, the following relations hold, i.e. $C_{1}(\mathcal{E}(\eta, 0)) \leq C_{1}\left(\mathcal{E}\left(\eta, s_{1}\right)\right) \leq \cdots \leq C_{1}\left(\mathcal{E}\left(\eta, s_{n}\right)\right) \leq$ $C_{1}(\mathcal{E}(\eta, 1)) \equiv C_{1}(\mathcal{D}(\eta))$ for $0 \leq s_{1} \leq \cdots \leq s_{n} \leq 1$. In other words the $\eta$-dependence of $C_{1}(\mathcal{E}(\eta, s))$ will be described by a continuous family of lines for intermediate value of $s$ in the range $[0,1]$, i.e. between the red $(s=1)$ and black $(s=0)$ extreme lines in Fig. 2. From these results it turns out that the presence of dephasing, on one hand, may increase the value of $\eta$ enhancing also $C_{1}$, but, on the other hand, increasing the amount of decoherence in the network (i.e., decreasing $s$ ), may reduce the value of $C_{1}$. Hence, a compromise of these two dephasing-induced effects could lead to a global enhancement of the classical capacity, as shown below.

Example: FMO-channel capacity.- Here, we will describe a concrete example of the results above. In particular, we will consider the transport dynamics of electronic excitations in a biological pigment-protein complex, called FMO (Fenna-Matthews-Olson) complex, involved in the early steps of photosynthesis in sulphur bacteria [10]. It is possible to describe this complex as a network of 7 chromophores or sites, represented as qubits. The coherent exchange of excitations between sites can be described by the Hamiltonian above plus local Lindblad terms that take into account the dephasing and dissipation caused by the surrounding environment - see Ref. [7] for more details about this model. Actually, the channel in Eq. 22 preserves the same form also in presence of dissipation, which will simply affect the values of $\eta$ and $s$. Moreover, the exciton energy is irreversibly transferred to the socalled reaction center, where it is immediately (irreversibly) converted into chemical energy. At each time $t$ (in a scale of order of ps), this evolution can be mapped as in Eq. (2), where $\eta$ is equal to the amount of excitations $p_{\operatorname{sink}}(t)$ in the reaction center with one excitation initially in site 1 , while $s=0$ since there is just a population transfer from the site 3 to the reaction center, i.e. the map is $\mathcal{E}\left(p_{\text {sink }}(t), 0\right)$. By choosing the parameters as in Ref. [7], we find that the classical capacity of the FMO complex dynamics, described as a quantum channel, $C_{1}\left(\mathcal{E}\left(p_{\text {sink }}(t), 0\right)\right)$, is remarkably enhanced in the presence of dephasing, especially after 1 ps - see Fig. 3. The dephasing enhanced classical capacity is due to the acceleration of transport in the network and may have been expected from the results of [5-7] as dephasing noise does not affect classical information. Dephasing noise however destroys quantum information and it is therefore not immediately evident that the quantum capacity may be enhanced by dephasing as well.

Quantum capacity.- The quantum capacity $Q$ refers, instead, to the coherent transmission of quantum information (measured in number of qubits), i.e. quantum states, through a quantum channel. It is more difficult to treat than the classical capacity discussed above and its explicit calculation is one of the basic issues in quantum information science. The single-use formula for the quantum capacity is obtained maximixing the coherent information [11], i.e.

$$
Q_{1}(\mathcal{E})=\max _{\rho \in \mathcal{H}}[S(\mathcal{E}(\rho))-S(\rho, \mathcal{E})]
$$

where the maximization is performed over all qubit states in the input Hilbert space $\mathcal{H}$. Here, $S(\rho, \mathcal{E})$ is the exchange entropy of the channel [1], describing the amount of information exchanged between the system and the environment after the noisy evolution, and is given by $S(\rho, \mathcal{E}) \equiv S(W)=$ $-\operatorname{Tr}\left[W \log _{2} W\right]$ with $W_{i j}=\operatorname{Tr}\left[A_{i} \rho A_{j}^{\dagger}\right]$. Note that $Q_{1}$ is usually a lower bound for $Q$ (which is maximized over many channel uses), since the coherent information is generally not additive. As discussed above, for simplicity, we will optimize numerically the coherent information over all possible input states $\rho_{A}$ as in (2). It turns out numerically that the expression to maximize is always decreasing in $|\gamma|^{2}$ for $\eta \geq 1 / 2$ and then it achieves the maximum value for $\gamma=0$; the remaining optimization in $p$ has been performed numerically and the results are shown in Fig. 2. In the case of $s=1$, the channel reduces to $\mathcal{D}(\eta)$, for which the coherent information can be proved to be additive (i.e., since it is a degradable channel [12]), and the optimization over the channel uses is not necessary. The behaviour of the quantum capacity $Q$ as a function of $\eta$ for $s=1$ has been investigated in Ref. [8]. Here, we generalize those results in the presence of dephasing, i.e. $s<1$, and find numerically the one-shot quantum capacity $Q_{1}$ as in Fig. 2. The additivity of the coherent information cannot be proved for $s<1$ but our results for $Q_{1}$ are of course a lower bound for the capacity $Q$ with entangled 


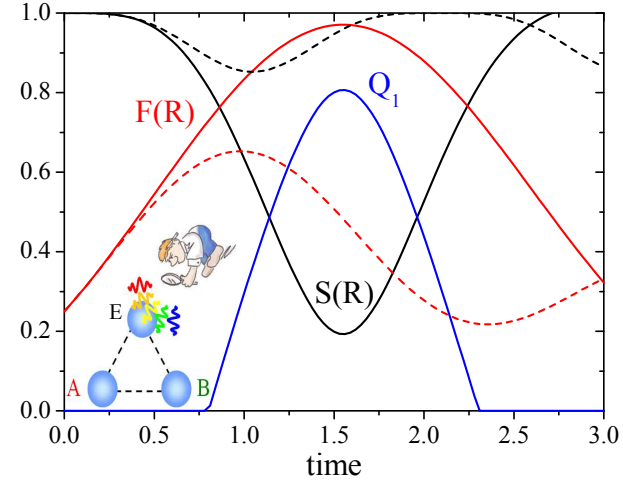

FIG. 4. Quantum capacity $Q_{1}$ for a 3 -site network vs. time. In absence of noise, $Q$ is always zero, while $Q_{1}$ can get values almost up to one in the presence of dephasing. Here, the hopping and dephasing rates are $v_{12}=v_{23}=v_{13}=1$, and $\gamma_{2}=50, \gamma_{1}=\gamma_{3}=0$. The corresponding channel entropy and fidelity are also shown in the noiseless (dashed) and dephasing (continuous line) case.

encodings. Although we have not computed the full quantum capacity rigorously and analytically, our lower bounds are sufficient to show the noise-assisted enhancement for the unrestricted quantum capacity as well. Indeed, because of the composition law $\mathcal{E}(\eta, s)=\mathcal{D}(\eta) \circ \mathcal{N}(s)$, the channel in (2) has always a vanishing $Q$ for $\eta \leq 1 / 2$ and for any $s$, since in this regime $\mathcal{D}(\eta)$ has $Q=0$ (being an anti-degradable channel) [8, 13]. Similar enhancement is observed in other figures of merit, i.e. channel fidelity $F(R)$ and entropy $S(R)$ [14, 15], which can be analytically derived for the map in (2), i.e. $F(R)=1 / 4(1+\eta+2 \sqrt{\eta s})$ and $S(R)=-\sum_{i=1}^{3} \lambda_{i} \log _{2} \lambda_{i}$, with $\lambda_{1}=(1-\eta) / 2, \lambda_{2,3}=1 / 4\left(1+\eta \pm \sqrt{4 \eta s+(1-\eta)^{2}}\right)$. A specific example of a 3 -qubit network is shown in Fig. 4. The presence of dephasing 'switches on' the channel capability of transmitting quantum information and the optimal rates can be very close to one (i.e., almost perfect state transfer), while $Q$ is exactly zero without dephasing. The intuitive reason for this behaviour is the fact that, in the noisefree case, quantum information progresses along two possible paths, thus being split and not arriving at the same time. This approximates a channel that splits the quantum information which in turn has vanishing quantum capacity. For strong dephasing the path via $E$ is blocked and direct transfer from $A$ to $B$ leads to the arrival of all quantum information at $B$. Noise assisted channel capacities may also be observed for larger networks and when all sites suffer dephasing, but the effect is most pronounced for non-uniform distribution of the noise. As final remark, notice that the dephasing can be induced by the presence of an eavesdropper, Eve, in the third site, in a quantum cryptographic scenario and, interestingly enough, it turns out that the eavesdropping operation is completely useless for Eve (the corresponding quantum capacity for the channel $A \rightarrow E$ remains exactly zero), but it does sensibly improve the Alice-Bob communication.

Conclusions and Outlook.- We have evaluated analytically and numerically the classical and quantum channel ca- pacities of a realistic communication network and showed that these optimal information transmission rates can be enhanced by applying some pure dephasing to the network. In particular, this allows us to reinterpret the observed dephasingassisted EET in photosynthetic complexes as an example of a quantum channel with a noise-enhanced classical capacity. Perhaps more surprisingly, we have shown that the presence of noise may lead to a finite quantum capacity where the noiseless system has vanishing capacity. As a result, not only the transmission rate of classical information can be assisted by noise but also the transmission of quantum information coded in quantum states. The possible relevance of this result in the context of quantum cryptography has been illustrated in a simple scenario. We expect these results to be easily generalizable to bosonic systems and to be valid for any Hamiltonian preserving the number of excitations, other forms of noise and also for non-Markovian evolutions. Finally, the three-site quantum network illustrating the fundamentals of our results could be experimentally investigated relatively easily by considering, for instance, quantum information platforms using trapped ions or cold atoms where forms of dephasing noise can be introduced in a controlled manner [16].

This work was supported by EPSRC grant EP/C546237/1, the EU STREP project CORNER, the EU Integrated project QAP, the Royal Society, a Marie Curie Fellowship, and a Alexander von Humboldt Professorship.

[1] M. Nielsen and I. Chuang, Quantum Computation and Quantum Information (Cambridge University Press, UK, 2000).

[2] C. Bennett and P. Shor, IEEE Trans. Inf. Theo. 44, 2724 (1998).

[3] M.B. Plenio and S.F. Huelga, Phys. Rev. Lett. 88, 197901 (2002); L. Hartmann et al., Phys. Rev. A. 74, 052304 (2006).

[4] C. Di Franco et al., Phys. Rev. A 77, 062337 (2008); G. Bowen and S. Mancini, Phys. Lett. A 321, 1 (2004); ibid. 352, 272 (2006).

[5] M. Mohseni et al., J. Chem. Phys. 129, 174106 (2008).

[6] M.B. Plenio and S.F. Huelga, New J. Phys. 10, 113019 (2008).

[7] F. Caruso et al., J. Chem. Phys. 131, 105106 (2009); P. Rebentrost et al., New J. Phys. 11, 033003 (2009) A. Chin et al., Eprint arXiv:0910.4153.

[8] V. Giovannetti and R. Fazio, Phys. Rev. A 71032314 (2005).

[9] A. S. Holevo, IEEE Trans. Inf. Theo. 44, 269 (1998); P. Hausladen et al., Phys. Rev. A 54, 1869 (1996); B. Schumacher and M.D. Westmoreland, ibid. 56, 131 (1997).

[10] H. Lee et al., Science 316, 1462 (2007); V. I. Prokhorenko et al., J. Phys. Chem. B 106, 9923 (2002); J. Adolphs and T. Renger, Biophys. J. 91, 2778, (2006).

[11] S. Lloyd, Phys. Rev. A 55, 1613 (1997); H. Barnum et al., Phys. Rev. A 57, 4153 (1998); I. Devetak, IEEE Trans. Inf. Theory 51, 44 (2005).

[12] I. Devetak and P.W. Shor, Comm. Math. Phys. 256, 287 (2005).

[13] F. Caruso and V. Giovannetti, Phys. Rev. A 74, 062307 (2006); Phys. Rev. A 76, 042331 (2007).

[14] W. Roga et al., J. Phys. A: Math. Theor. 41, 035305 (2008).

[15] D. Kretschmann and R.F. Werner, New J. Phys. 6, 26 (2004).

[16] A. Bermudez et al., E-print arXiv:1002.3748 (2010). 